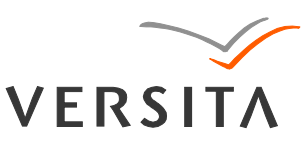

GEOCHRONOMETRIA 41(4) 2014: 327-333

DOI 10.2478/s13386-013-0174-x

Available online at

www.springerlink.com

\title{
PERFORMANCE TESTS USING THE Lexsyg LUMINESCENCE READER
}

\author{
JOHANNA LOMAX ${ }^{1}$, SEBASTIAN KREUTZER ${ }^{2,1}$ and MARKUS FUCHS ${ }^{1}$ \\ ${ }^{I}$ Department of Geography, Justus-Liebig-University Giessen, Senckenbergstr. 1, 35390 Giessen, Germany. \\ ${ }^{2}$ IRAMAT-CRP2A, Université Bordeaux Montaigne, Maison de l'Archéologie, Esplanade des Antilles, 33607 Pessac Cedex, France
}

Received 10 March 2014

Accepted 4 August 2014

\begin{abstract}
This study presents test measurements on a Lexsyg luminescence reader, acquired by the Giessen luminescence group. The reader is of the type Standard, hence designed for routine determination of palaeodoses using quartz or K-feldspars. The tests include measurements of the stimulation powers, preheat temperatures, OSL- and TL-curves, as well as dose recovery tests and calibration measurements using highly sensitised calibration quartz. A comparison of $D_{\mathrm{e}}$ values determined using a Lexsyg reader with single grain $D_{\mathrm{e}}$-values, which have been previously obtained on a Risø reader, is also presented. The results imply that the Lexsyg reader is a highly reliable measurement device with high reproducibility, yielding instrumental uncertainties of around $0.3 \%$.
\end{abstract}

Keywords: dosimetry, instrumentation, reproducibility, luminescence dating.

\section{INTRODUCTION}

Since July 2012, the Giessen luminescence laboratory runs a recently developed luminescence reader, termed Lexsyg Standard, designed by the German company Freiberg Instruments (Richter et al., 2013). Readers of this type are designed for standard optically stimulated and infrared stimulated luminescence (OSL and IRSL) measurements. They are typically equipped with a photomultiplier tube and a six-position filter wheel for signal detection, a $\beta$-source for irradiation, two to three light sources for stimulation and a heater plate for preheat procedures and thermoluminescence (TL) measurements. The standard reader type can be upgraded to a reader of the type "research", by adding e.g. a spectrometer, a radiofluorescence unit, a solar simulator or an EM-CCD camera. One principle difference compared to existing systems is that only one aliquot occupies the measurement chamber at the time of measurement, preventing optical crosstalk and minimising cross-irradiation. A further difference to many existing luminescence readers is the fully automated filter changer, which allows applying different detection windows during measurement. However, Risø now also offers an automated filter changer. The Lexsyg Standard at the Giessen laboratory was mainly acquired for routine measurements of equivalent doses $\left(D_{\mathrm{e}}\right)$ for palaeoenvironmental dating studies. Being one of the first of its type, the luminescence reader requires thorough testing of its performance. This paper reports on test measurements, providing information on the stability of the stimulation sources and heater plate, and on the instrumental uncertainty. Dose recovery tests (DRT) and natural dose measurements of samples with "known" $D_{\mathrm{e}}$ values are also shown to demonstrate the performance. The results provide important guidelines for the use of the Lexsyg luminescence readers for $D_{\mathrm{e}}$ determination.

Corresponding author: J. Lomax

e-mail: Johanna.lomax@geogr.uni-giessen.de 


\section{TECHNICAL SETUP}

A detailed description of the technical setup of the Lexsyg luminescence reader was recently provided by Richter et al. (2013). In the following, the specific technical setup of the Giessen Lexsyg is described. The setup may vary from descriptions given in Richter et al. (2013) and from other Lexsyg readers in use.

\section{Instrumental details of components}

Stimulation is performed with a 15 position stimulation wheel, with 10 positions occupied by green LEDs and 5 positions occupied by IR laser diodes. The maximum emission of the green LEDs is at $525 \Delta 25 \mathrm{~nm}$, the bandwidth being confined by optical filters in front of the LEDs (Schott OG 515 (3 mm) + Semrock HC530/43). The 10 LEDs deliver a maximum of $70 \mathrm{~mW} / \mathrm{cm}^{2}$ to the disc surface. The IR laser diodes emit at $850 \Delta 3 \mathrm{~nm}$ and deliver a maximum power density of $400 \mathrm{~mW} / \mathrm{cm}^{2}$ to the disc surface. The optical power of every green LED and IR laser diode is constantly monitored and regulated individually during stimulation.

Signals are detected by a Hamamatsu photomultiplier tube (H7360), with an optimum quantum efficiency centred at $375 \mathrm{~nm}$, and enhanced detection efficiency in the visible range $(300-650 \mathrm{~nm})$. Before reaching the photomultiplier, the light passes through optical filters. These are placed in a six-position filter-wheel, and filters can be changed during the measurement cycle. Currently, the Lexsyg in Giessen is equipped with two UV-filters for quartz measurements centred on $340 \mathrm{~nm}$ and $380 \mathrm{~nm}$ (Semrock HC340/26 (5 mm) + BG3 (3 mm) and Semrock HC377/50 (5 mm) + BG3 (3 mm)), a blue interference filter for feldspar measurements centred on $410 \mathrm{~nm}$ (Sem- rock HC414/46 nm (3.5 mm) + BG39 (3 mm)), a broad band blue filter (Schott KG3, BG25 and BG39, each $3 \mathrm{~mm}$ ), a red filter for RTL measurements (Chroma $620 / 60(3.5 \mathrm{~mm})+\mathrm{KG} 3(6 \mathrm{~mm}))$ and a yellow filter (Semrock HC575/50 (5 mm) + KG3 (3 mm)). Fig. 1 shows filter transmission spectra (www.semrock.com and www.schott.com) of the most relevant filters used for routine quartz and $\mathrm{K}$-feldspar luminescence measurements, and emission spectra/emission lines of the stimulation sources. Also shown are OSL and IRSL emission spectra of sedimentary quartz and K-feldspar samples, taken from Huntley et al. (1991).

Irradiation is provided by a $\mathrm{Sr}-90 / \mathrm{Y}-90 \quad \beta$-source which consists of 17 single $\beta$-sources. Its activity is $1.9 \mathrm{GBq}$ and it delivers a dose rate of $\sim 0.135 \pm 0.003 \mathrm{~Gy} / \mathrm{s}$ to coarse grain quartz samples $(90$ $125 \mu \mathrm{m})$ when using stainless steel cups. This $\beta$-source differs from the ring-source described in Richter et al. (2012), as it is a non-ring type $\beta$-source and not suitable for RF-measurements. According to measurements performed by the producer of the source (Pintaske Consulting), its dose rate varies up to $15 \%$ over a disc diameter of $8 \mathrm{~mm}$, thus its homogeneity is significantly lower than for the ring-type $\beta$-source $(<3 \%$, Richter et al., 2012). Further homogeneity tests of the source have so far not been carried out at the Giessen luminescence laboratory, although previous studies have shown the importance of such tests (e.g., Ballarini et al., 2006; Lapp et al., 2012). However, as no single grain measurements are carried out on the Giessen Lexsyg, source homogeneity is of lesser importance than for single grain studies.

A detailed description of the heater plate and the arrangement of components can be found in Richter et al. (2013).
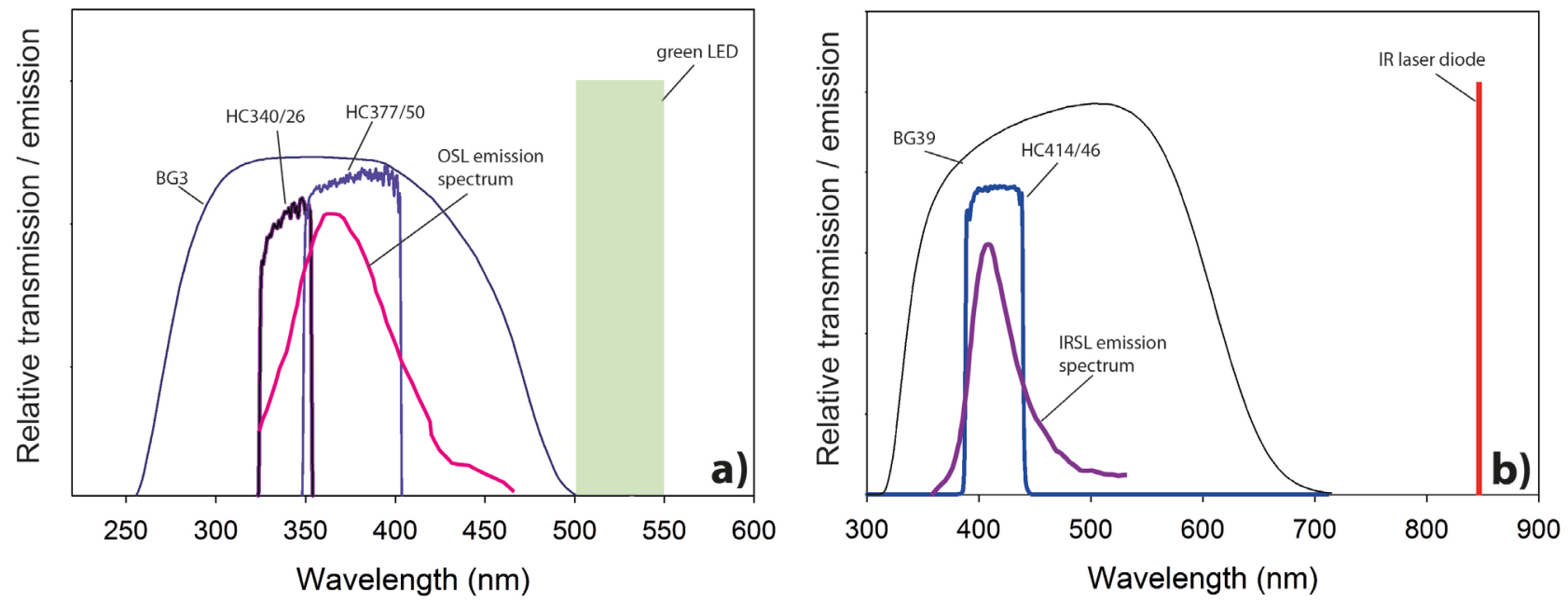

Fig. 1. Transmission spectra of most relevant filters for a) routine quartz luminescence measurements and b) routine K-feldspar luminescence measurements. Also shown are emission bands/lines of the stimulation sources, and OSL and IRSL emission spectra of quartz and feldspar, taken from Huntley et al. (1991). 


\section{Software control}

Sequence writing and reader control is carried out using the Freiberg Instruments software "LexStudio 2" operating under Microsoft Windows ${ }^{\mathrm{TM}}$. A detailed software description is beyond the scope of this contribution, but some relevant aspects are highlighted.

LexStudio 2 provides direct access to the hardware components within the reader. This concept allows a high flexibility for individual and complex hardware configurations. Measurement results can be stored as *.csv (comma-separated values), *.bin/*.binx (binary Risø reader file format) or *.xsyg (XML based file format developed by Freiberg Instruments) files. The latter one provides full access to all recorded measurement values. For example, for each TL curve three different values are stored: (1) The predefined heating ramp curve (time $v s$. temperature), (2) the real measured heating ramp (time vs. temperature) recorded by the heating element itself and (3) the TL count values (time vs. counts) from the PMT. Similar curves are recorded for irradiation (time $v s$. $\beta$-source on/off) or optical stimulation (predefined stimulation power, real stimulation power, count values). For the latter, also the measured stimulation temperature is recorded (also for irradiation, if this is performed at an elevated temperature). All monitored curves are graphically displayed during each measurement. The close monitoring of every measured value (including stimulation powers and preheat/stimulation temperatures) allows a direct control on the quality of each measurement step.

\section{TEST MATERIAL}

For some of the tests shown in this paper, sedimentary quartz and K-feldspar samples are used. Geological derivation and treatment of these test samples are described in the following. For coarse grain quartz measurements, Risø calibration quartz (labelled "Risø calQ" in the following; $90-125 \mu \mathrm{m}$ ) was used. Originally derived from a Scandinavian sedimentary deposit, the quartz was annealed at high temperatures, in order to remove the natural dose and to sensitise the sample. A $\gamma$-dose of 5 Gy was administered to this sample (Vandenberghe, 2004). Sample BT379 is a fine grained quartz $(4-11 \mu \mathrm{m})$, composed of Saharan dust and was collected on Lanzarote. The sample was annealed and sensitised at high temperatures and given a $\gamma$-dose of $2.88 \mathrm{~Gy}$. For measurements of coarse grain K-feldspar, sample NWC1 $(100-200 \mu \mathrm{m})$ was used. Derived from a dune sand within the western Murray Basin (SE Australia), this sample has a natural dose of $>300 \mathrm{~Gy}$. Further details on the sedimentary setting can be found in Lomax et al. (2011). For the test measurements, bleached and $\beta$-irradiated subsamples were used. In case $D_{\mathrm{e}}$ values were determined, the measurements are based on the Single-Aliquot RegenerativeDose (SAR) protocol of Murray and Wintle (2000).

\section{PERFORMANCE OF OSL/IRSL STIMULATION SOURCES}

\section{Monitoring of stimulation power}

During measurement, a photodiode measures the optical power output of every individual stimulation device. This monitoring and logging of power densities (via *.csv or *.xsyg files) is a powerful tool to ensure stable stimulation conditions. Graphs of the power monitoring are shown in Fig. 2. It can be observed that both green and IR stimulation sources exhibit very stable stimulation powers. The typical standard deviation on the power density from their mean values over the time period of stimulation is $0.1 \%$, equally for the green and the IR stimulation.

\section{OSL and IRSL decay curves}

Fig. 3a shows a comparison of quartz OSL decay curves, generated with green LEDs from the Lexsyg reader and blue LEDs from a Risø TL-DA20 reader
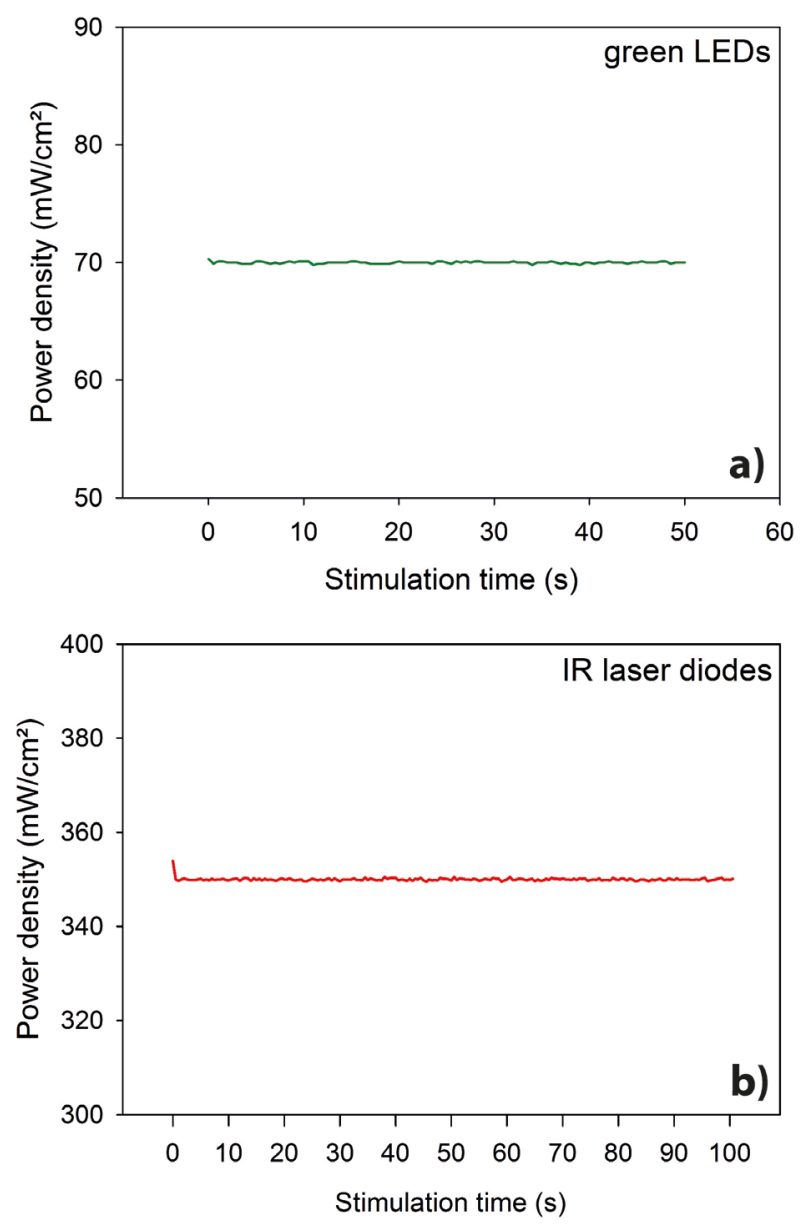

Fig. 2. Stimulation power logs using a) the green LEDs at $70 \mathrm{~mW} / \mathrm{cm}^{2}$, and b) the IR laser diodes at $350 \mathrm{~mW} / \mathrm{cm}^{2}$. 
(BOKU Vienna). The green stimulation source was set at a power density of $70 \mathrm{~mW} / \mathrm{cm}^{2}$, whereas the blue LED power density was set at $c a .41 \mathrm{~mW} / \mathrm{cm}^{2}$. Green stimulated OSL signals were filtered through the $380 \mathrm{~nm}$ filter (see Section 2), whereas the blue stimulated signal through a Hoya U-340 $(7.5 \mathrm{~mm})$ filter. In both cases, the stimulation temperature was set to $125^{\circ} \mathrm{C}$, and OSL measurements were preceded by preheats of $220^{\circ} \mathrm{C}$ $(10 \mathrm{~s})$. As expected, the blue stimulated OSL decays faster compared to the green stimulated OSL, due to the higher energy of the stimulation wavelength (cf. Spooner, 1994; Duller and Bøtter-Jensen, 1996; Bailey et al., 2011). This results in a slightly disadvantageous signal to noise ratio (SNR) when using green stimulation. On the other hand, signals can be enhanced compared to blue stimulation by placing the detection window directly onto the peak emission of quartz at $c a .380 \mathrm{~nm}$ (Martini and Galli, 2007, see also Fig. 1). If a higher SNR is preferred, this can theoretically be achieved by increasing the stimulation temperature. Bailey et al. (2011) recommend stimulation temperatures of $130-160^{\circ} \mathrm{C}$ in case of green stimulation. A further advantage of green stimulation, according to Singarayer and Bailey (2003) and Bailey et al. (2011), is a better isolation of the fast component.

Fig. $3 \mathbf{b}$ shows typical IRSL decay curves of feldspar coarse grains, measured with a Risø reader and the Lexsyg. The Risø reader (BOKU Vienna) uses an array of 24 infrared LEDs, emitting at $870 \mathrm{~nm}$, and delivering $c a .143 \mathrm{~mW} / \mathrm{cm}^{2}$ to the sample. In the Lexsyg, power densities were set to $300 \mathrm{~mW} / \mathrm{cm}^{2}$. In both readers, luminescence light was filtered through $c a .410 \mathrm{~nm}$ interference filters (see Section 2) and stimulation temperatures were set to $50^{\circ} \mathrm{C}$. Measurements were preceded by preheats at $250^{\circ} \mathrm{C}(60 \mathrm{~s})$. The Lexsyg IR stimulation shows a faster decay, probably owing to the higher power density of the laser compared to the IR-LEDs. It should further be noted that high laser powers, as the case for the Lexsyg reader, may induce a temperature rise on the sample disc, which in turn may increase the decay rate. However, for feldspars - in contrast to quartz - it is not of primordial importance to enhance decay rates for better SNRs. Further implications, e.g. for fading rates, also need to be investigated further.

\section{STABILITY OF HEATER PLATE AND TL- PERFORMANCE}

Temperatures on the heater plate are routinely monitored within the heating element by measuring the electric resistance. Fig. 4a shows such a monitoring, collected during a TL measurement (preheat $260^{\circ} \mathrm{C}$ for $10 \mathrm{~s}$, heating rate of $5^{\circ} \mathrm{C} / \mathrm{s}$ ), an IRSL-50 and a post IR IRSL-225 measurement. According to the log-values, temperature variations are minimal (standard deviation of $0.5 \%$ at $50^{\circ} \mathrm{C}$ and $0.2 \%$ at $225^{\circ} \mathrm{C}$ ). It needs to be considered though that heat from the heater plate needs to reach the sample through relatively thick $(0.5 \mathrm{~mm})$ cups, made of aluminium, nickel or stainless steel. Therefore a considerable lag of temperature between heater plate and sample is expected. It is thus advisable to wait with stimulation for at least $10 \mathrm{~s}$ after the heater plate reaches the respective stimulation temperature. This time between beginning of stimulation and detection after reaching the final temperature on the heater plate can be chosen individually by the user. Fig. $4 \mathbf{b}$ shows seven TL curves of the calibration quartz Risø calQ, recorded during a common SAR-cycle after each test dose of $2.6 \mathrm{~Gy}$. The TL curves are very similar in shape and peak position, indicating - besides uniform luminescence characteristics throughout the SAR cycle - that heating conditions are very stable.
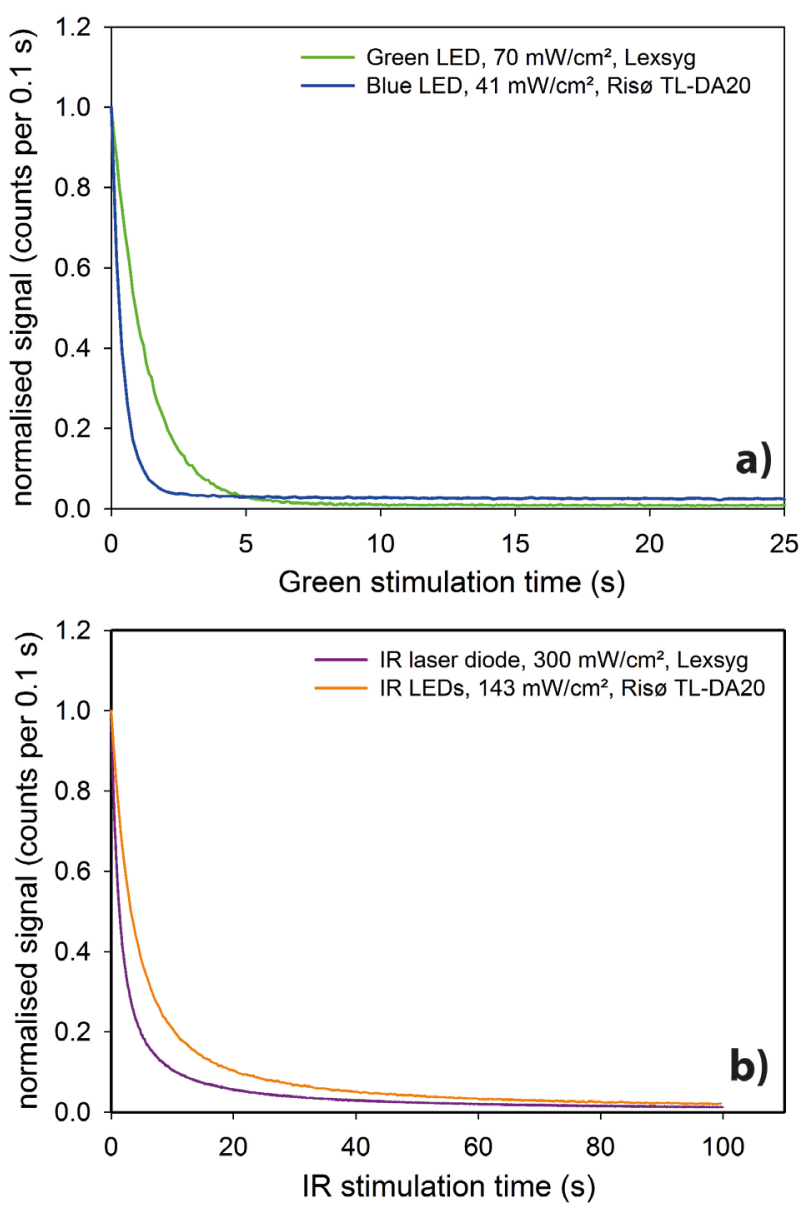

Fig. 3. Normalised OSL and IRSL decay curves, achieved with a) green and blue LEDs using the Risø calibration quartz. Green stimulation was performed on the Lexsyg (University Giessen), blue stimulation on a Risø TL-DA20 (BOKU Vienna), and b) IR laser diodes and IR LEDs on the K-feldspar sample NWC1. IR laser diode stimulation was performed on the Lexsyg (University Giessen), IR-LED stimulation on a Risø TL-DA20 (BOKU Vienna). 

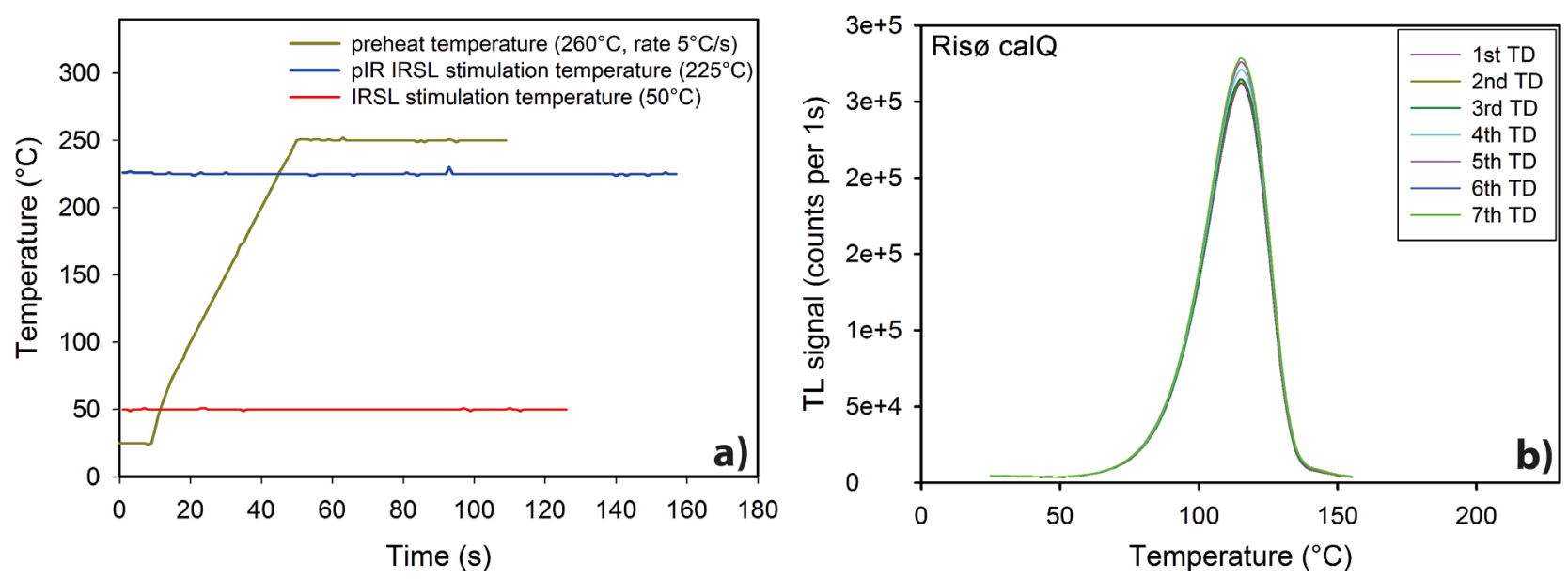

Fig. 4. TL-performance of the Lexsyg luminescence reader, a) monitored "real" stimulation and preheat temperatures, b) seven consecutive $T L$ measurements following the test doses (TD) of one SAR cycle.

\section{REPRODUCIBILITY}

A first check on the reproducibility of the Lexsyg reader was performed by measuring 30 repeated doses on one aliquot of the coarse grain calibration quartz Risø calQ, using the green LED stimulation source. A mask size of $8 \mathrm{~mm}$ was used to produce bright luminescence signals and little uncertainty due to counting statistics. The sample was bleached to zero in the Lexsyg reader (green LED, $70 \mathrm{~mW} / \mathrm{cm}^{2}, 80 \mathrm{~s}, 125^{\circ} \mathrm{C}$ ). Subsequently, the aliquot was given 32 regeneration doses of $2.6 \mathrm{~Gy}$. The preheat temperature was set to $220^{\circ} \mathrm{C}$. Signals were integrated over the first $0.5 \mathrm{~s}$, subtracted by a background signal integrated over the last $20 \mathrm{~s}$ of stimulation. Due to non-linear sensitivity changes, signals were normalised by treating every second signal as test dose $\left(T_{\mathrm{x}}\right)$ and calculating $16 L_{\mathrm{x}} / T_{\mathrm{x}}$ values. These $L_{\mathrm{x}} / T_{\mathrm{x}}$ values exhibit a relative standard deviation (RSD) of $0.34 \%$, of which $0.20 \%$ can be accounted for by counting statistics. The latter number was derived from the average relative uncertainty of each individual $D_{\mathrm{e}}$ value, calculated with the program Analyst (Version 3.24), based on the equation given in Galbraith (2002). Further details on the error estimation used in the Analyst are given in Duller (2007). The remaining uncertainty of $0.27 \%\left(\sqrt{0.34^{2}-0.20^{2}}\right)$ will mainly be the result of instrumental variations. The result of this reproducibility test is shown in Fig. 5.

Furthermore, dose recovery tests (DRT) and calibration measurements were carried out, focussing on quartz and green stimulation. Results of these tests can be found in Table 1 and Fig. 6. As material, the Risø calQ for the coarse grain measurements and sample BT379 for the fine grain measurements was used. In a first step, a dose recovery test (DRT) was performed. The material was bleached in the luminescence reader with the green LED $\left(70 \mathrm{~mW} / \mathrm{cm}^{2}, 50 \mathrm{~s}, 125^{\circ} \mathrm{C}\right)$, and given a beta dose of $5 \mathrm{~Gy}$ (Risø calQ) or 3.6 Gy (BT 379) using the $\beta$-source of the Lexsyg reader. In the following, a standard SAR protocol (Murray and Wintle, 2000) was applied, with preheat and cutheat temperatures at $220^{\circ} \mathrm{C}$ and $160^{\circ} \mathrm{C}$. Stimulation was carried out with the green LEDs, and signals were detected with the $380 \mathrm{~nm}$ filter. Signals were integrated over the first $0.5 \mathrm{~s}$ of stimulation and subtracted by the last $10 \mathrm{~s}$ of stimulation as background signal. The resulting dose recovery ratios were very close to unity, and

Table 1. Results and statistical parameters of dose recovery tests and calibration measurements using calibration quartz material. Overdispersion $(O D)$ was calculated by incorporating and instrumental error of $0.3 \%$.

\begin{tabular}{ccccccccc}
\hline Sample & $\begin{array}{c}\text { Type of mea- } \\
\text { surement }\end{array}$ & Stimulation & $\begin{array}{c}\text { Dose recov- } \\
\text { ery ratio }\end{array}$ & $\mathbf{n}$ & $\begin{array}{c}\text { RSD } \\
(\%)\end{array}$ & $\begin{array}{c}\text { Average error from } \\
\text { counting statistics } \\
(\%)\end{array}$ & $\begin{array}{c}\text { Overdis- } \\
\text { persion } \\
(\%)\end{array}$ & $\begin{array}{c}\text { Additional error } \\
\text { from Y-irradiation } \\
(\%)\end{array}$ \\
\hline $\begin{array}{c}\text { Ris } \varnothing \mathrm{calQ} \\
(90-125 \mu \mathrm{m})\end{array}$ & Calibration & Green LED & & 20 & 2.1 & 0.6 & 2.0 & 1.8 \\
\hline $\begin{array}{c}\text { Ris } \varnothing \mathrm{calQ} \\
(90-125 \mu \mathrm{m})\end{array}$ & DRT & Green LED & 1.00 & 19 & 1.0 & 0.6 & 0.6 & \\
\hline $\begin{array}{c}\text { BT 379 } \\
(4-11 \mu \mathrm{m})\end{array}$ & Calibration & Green LED & & 12 & 1.7 & 1.2 & 0.0 & 1.6 \\
\hline $\begin{array}{c}\text { BT 379 } \\
(4-11 \mu \mathrm{m})\end{array}$ & DRT & Green LED & 0.99 & 10 & 0.7 & 0.8 & 0.0 & \\
\hline
\end{tabular}




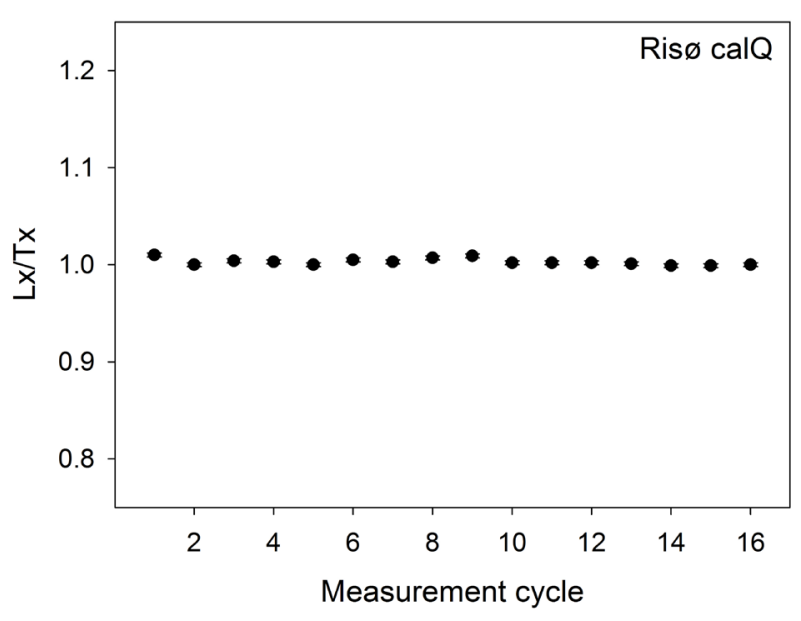

Fig. 5. Reproducibility test of the coarse grain quartz calibration sample. 32 doses of $2.6 \mathrm{~Gy}$ were administered and $16 L_{x} / T_{x}$ values were calculated from the OSL signals, which display a relative standard deviation of $0.3 \%$.

RSDs ranged from $0.7 \%$ (fine grained sample) to $1.0 \%$ (coarse grained samples (Table 1, Fig. 6a, 6c). Assuming ideal performance of the measurement protocol, these variations will be the result of several sources: the instrumental uncertainty of the Lexsyg reader, counting statistics, the error on the curve fit and error increment through a slightly non-linear growth behaviour (e.g., Duller et al., 2000; Thomsen et al., 2005; Duller, 2007). For the coarse grain sample, the resulting overdispersion (OD) of the DRT is close to zero when including an instrumental uncertainty of $0.3 \%$, thus the main sources of errors - uncertainty through counting statistics $(0.6 \%)$ and instrumental uncertainty account for the majority of observed $D_{\mathrm{e}}$ variations. For the fine grain sample, also measured with the green LED, the total uncertainty (RSD) of $0.7 \%$ can entirely be accounted for by counting statistics $(0.8 \%)$, without even including an instrumental error.

In a second step, the known gamma doses of the calibration sources were measured, using the same SAR protocol as described above. When performing these calibration measurements, relative standard deviations between $1.7 \%$ and $2.1 \%$ were observed (Table 1, Fig. $\mathbf{6 b}$, 6d). Thus, it is once again noted that an additional source of error is introduced when the samples are irradiated externally with a $\gamma$-source. This effect was already recognised by Cunningham et al. (2011) and Thomsen et al. (2005), and occurs despite the fact that $\gamma$-irradiation in sand sized grains should be relatively uniform considering the high range of $c a .30 \mathrm{~cm}$ in quartz. Cunningham et al. (2011) suspected that the different orientation of the grains with respect to the radiation field and varying dose attenuation within the grains may account for this effect. In the present study, this additional source of uncertainty can be quantified with $1.5-1.8 \%$.
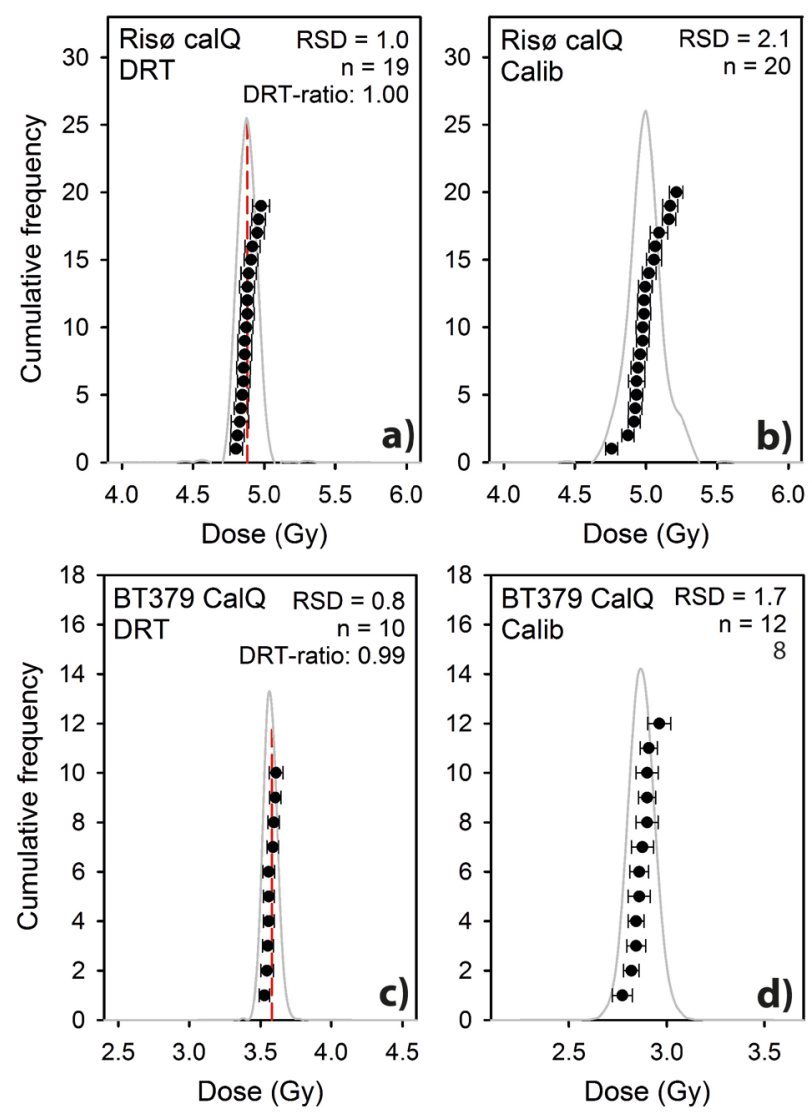

Fig. 6. De distributions of test measurements using "ideal" sample material (highly sensitised calibration quartz) with the following configurations: a) dose recovery test (DRT) of sample Risø calQ (coarse grain), b) $\gamma$-dosed sample Risø calQ, c) DRT of sample BT379 (fine grain), d) $y$-dosed sample $B T 379$ ( $R S D=$ relative standard deviation).

\section{DOSE MEASUREMENTS OF SAMPLES WITH "KNOWN" DOSES}

In a further test on the reliability of the new Lexsyg reader, $D_{\mathrm{e}}$ values determined in a Risø and in the Lexsyg reader were compared to each other. For this purpose a dune section from the western Murray Basin (Lomax et al., 2011) was chosen. This section showed comparably low OD and was considered as one of the few sections in the investigation area which was either not affected by bioturbation or where sand layers of the same age were mixed by bioturbation (hence not noticeable in the $D_{\mathrm{e}}$ distributions). The $D_{\mathrm{e}}$ determined in the Risø reader was carried out on quartz single grains, using a green laser for stimulation and grain sizes from $200-250 \mu \mathrm{m}$. Preheat and cutheat temperatures were set to $220^{\circ} \mathrm{C}$ and $160^{\circ} \mathrm{C}$. In case of the Lexsyg measurements, multiple grain aliquots ( $2 \mathrm{~mm}$ diameter) were used. The grain sizes ranged from $100-150 \mu \mathrm{m}$, and preheat and cutheat temperatures were set to $10^{\circ} \mathrm{C}$ higher $\left(230^{\circ} \mathrm{C}\right.$ and $\left.170^{\circ} \mathrm{C}\right)$, to account for the higher thermal inertia in the Lexsyg steel cups. The stimulation temperature was set to $125^{\circ} \mathrm{C}$ in both readers. The 
results of the comparison are shown in Fig. 7 and indicate that the doses are in very good agreement. However, this approach was just a first step to test the performance of the Lexsyg. In the future, these tests further need to include known aged samples (e.g., comparison with ${ }^{14} \mathrm{C}$ dated samples) and also with $D_{\mathrm{e}}$ values which were obtained with blue stimulation.

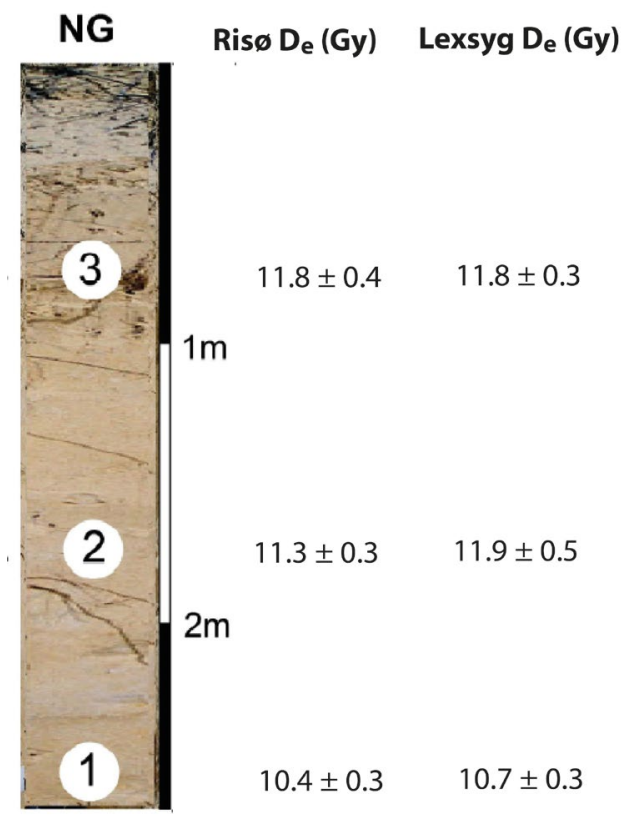

Fig. 7. Dune section of a parabolic dune in the Murray Basin (SEAustralia), and mean $D_{e}$ values obtained with a Risø reader on single grains of quartz (green laser stimulation) (Lomax et al., 2011) and with the Lexsyg reader on small multiple grain aliquots of quartz (green LED stimulation).

\section{CONCLUSIONS}

Performance tests on the Giessen Lexsyg luminescence reader have shown that high quality measurements for $D_{\mathrm{e}}$ determination can be performed on this device. Instrumental errors are minimal (around $0.3 \%$ ), owing to very stable stimulation and heating conditions. Furthermore, measurement parameters such as stimulation powers and preheat/stimulation temperatures are constantly monitored for quality control. A comparison between $D_{\mathrm{e}}$ values obtained on a Risø reader and on the Lexsyg yielded excellent agreement. However, further tests, e.g. comparing $D_{\mathrm{e}}$ values derived from green and blue stimulation need to be performed in the future.

\section{ACKNOWLEDGEMENTS}

The authors thank Andreas Richter and Dr. Kay Dornich (Freiberg Instruments) for constructive comments and help on the manuscript.

\section{REFERENCES}

Bailey R, Yukihara E and McKeever S, 2011. Separation of quartz optically stimulated luminescence components using green (525 nm) stimulation. Radiation Measurements 46(8): 643-648, DOI 10.1016/j.radmeas.2011.06.005.

Ballarini M, Wintle AG and Wallinga J, 2006. Spatial variation of dose rate from beta sources as measured using single grains. Ancient TL 24(1): 1-7.

Cunningham AC, Wallinga J and Minderhoud PSJ, 2011. Expectations of scatter in equivalent-dose distributions when using multi-grain aliquots for OSL dating. Geochronometria 38(4): 424-431, DOI 10.2478/s13386-011-0048-z.

Duller GAT, 2007. Assessing the error on equivalent dose estimates derived from single aliquot regenerative dose measurements. $A n$ cient TL 25(1): 15-24.

Duller GAT and Bøtter-Jensen L, 1996. Comparison of optically stimulated luminescence signals using different stimulation wavelengths. Radiation Measurements 26(4): 603-609, DOI 10.1016/1350-4487(96)00026-1.

Duller GAT, Bøtter-Jensen L and Murray AS, 2000. Optical dating of single sand-sized grains of quartz: sources of variability. Radiation Measurements 32(5-6): 453-457, DOI 10.1016/S13504487(00)00055-X.

Galbraith RF, 2002. A note on the variance of a background-corrected OSL count. Ancient TL 20: 49-51.

Huntley DJ, Godfrey-Smith DI and Haskell EH, 1991. Light-induced emission spectra from some quartz and feldspars. nternational Journal of Radiation Applications and Instrumentation. Part D. Nuclear Tracks and Radiation Measurements 18(1-2): 127-131, DOI 10.1016/1359-0189(91)90104-P.

Lapp T, Jain M, Thomsen KJ, Murray AS and Buylaert JP, 2012. New luminescence measurement facilities in retrospective dosimetry. Radiation Measurements 47(9): 803-808, DOI 10.1016/j.radmeas.2012.02.006.

Lomax J, Hilgers A and Radtke U, 2011. Palaeoenvironmental change recorded in the palaeodunefields of the western Murray Basin, South Australia - New data from single grain OSL-dating. Quaternary Science Reviews 30(5-6): 723-736, DOI 10.1016/j.quascirev.2010.12.015.

Martini M and Galli A, 2007. Ionic mechanisms in the optically stimulated luminescence of quartz. Physica Status Solidi: Current Topics in Solid State Physics 4(3): 1000-1003, DOI $10.1002 /$ pssc. 200673862.

Murray AS and Wintle AG, 2000. Luminescence dating of quartz using an improved single-aliquot regenerative-dose protocol. Radiation Measurements 32(1): 57-73, DOI 10.1016/S1350-4487(99)00253$\mathrm{X}$.

Richter D, Pintaske R, Dornich K and Krbetschek M, 2012. A novel beta source design for uniform irradiation in dosimetric applications. Ancient TL 30(2): 57-63.

Richter D, Richter A and Dornich K, 2013. Lexsyg - A new system for luminescence research. Geochronometria 40(4): 220-228, DOI 10.2478/s13386-013-0110-0.

Singarayer JS and Bailey RM, 2003. Further investigations of the quartz optically stimulated luminescence components using linear modulation. Radiation Measurements 37(4-5): 451-458, DOI 10.1016/S1350-4487(03)00062-3.

Spooner NA, 1994. On the optical dating signal from quartz. Radiation Measurements 23(2-3): 593-600, DOI 10.1016/13504487(94)90105-8.

Thomsen KJ, Murray AS and Bøtter-Jensen L, 2005. Sources of variability in OSL dose measurements using single grains of quartz. Radiation Measurements 39(1): 47-61, DOI 10.1016/j.radmeas.2004.01.039.

Vandenberghe D, 2004. Investigation of the optically stimulated luminescence dating method for application to young geological sediments. PhD Thesis, Ghent University: 358pp.

http://www.semrock.com/filters.aspx. Accessed on 01.02.2014.

http://www.schott.com/advanced_optics/english/products/opticalfilters/index.html. Accessed on 01.02.2014. 